Proceedings of the 2012 Winter Simulation Conference

C. Laroque, J. Himmelspach, R. Pasupathy, O. Rose, and A. M. Uhrmacher, eds.

\title{
EFFECTIVE CROWD CONTROL THROUGH ADAPTIVE EVOLUTION OF AGENT-BASED SIMULATION MODELS
}

\author{
$\mathrm{Nan} \mathrm{Hu}$ \\ School of Computer Engineering \\ Nanyang Technological University \\ Singapore, SINGAPORE
}

\author{
James Decraene \\ Institute of High Performance Computing \\ Agency for Science, Technology and Research \\ Singapore, SINGAPORE
}

\author{
Wentong Cai \\ School of Computer Engineering \\ Nanyang Technological University \\ Singapore, SINGAPORE
}

\begin{abstract}
We report an approach to achieve effective crowd control strategies through adaptively evolving an agentbased model of Crowd Simulation for Military Operations (COSMOS). COSMOS is a complex system simulation platform developed to address challenges posed by the Military Operations in Urban Terrains (MOUT). Modeling and simulating soldiers' tactical behaviors in MOUT scenarios is challenging due to the complex and emerging behaviors of crowds and large parameter space of the models. Consequently, it is difficult to search for effective crowd control strategies through tuning the model parameters manually. We employ an adaptive evolutionary computation approach, using the Complex Adaptive Systems Evolver (CASE), to address this challenge. Specifically, we conduct experiments using a "building-protection" scenario, where the operation plans of soldier agents are adaptively evolved to best control a crowd. The results suggest this approach using agent-based simulation and evolutionary computation techniques is promising for the study of complex military operations.
\end{abstract}

\section{INTRODUCTION}

Crowd control becomes increasingly important in Military Operations in Urban Terrain (MOUT) such as peace keeping, riot control, disaster management, emergency evacuation, and rescue operations (Larson 1981; Desch 2001; Grieger 2004). Given the military challenges and risks imposed by the crowds, Modeling \& Simulation (M\&S) techniques, in particular Agent-Based Simulations (ABS), are widely used to mimic various military missions to study the complex interactions of individual people in a crowd (Bonabeau 2002; Laclavk et al. 2012). ABS enables the study of consequences of actions that soldiers may carry out in the crowd control process (e.g., patrol path, commands and operations performed by the soldiers). Through the analysis of simulation outcomes for a particular scenario, the course of actions which results in the most effective crowd control for MOUT can be identified. This will effectively provide decision support to military analysts in operational plan and tactics development.

However, this operational analysis usually requires tens of thousands of simulations due to the complex behaviors emerging from the agent interactions, the stochastic nature of the model and the large number of parameters. For example, in a crowd control simulation, it is difficult to manually tune soldiers' parameters to obtain effective crowd control strategy even with a specific profile of the crowds. To utilize such ABS to conduct military operations research, it is thus important to apply an adaptive approach to automate the model generation and evaluation process. In a typical MOUT scenario, the objectives of the soldiers are 


\section{Hu, Decraene, and Cai}

usually clear and thus can be defined explicitly according to the purpose of the mission (Larson 1981). For example, in a building protection scenario, a good crowd control strategy would result in fewer people entering the building or their emotional status under control (e.g., the average anger level of the crowds is under certain threshold). From an "optimization" perspective, such scenarios provide clearly defined objectives, which can be formulated explicitly. In this paper, we propose to use evolutionary computation techniques to adaptively evolve some simulation model parameters to optimize such pre-defined objectives.

An agent-based simulation model to generate believable and realistic crowd behaviors needs to address two important components: the decision-making process and the emotion system. Naturalistic decision making (NDM) is argued to be an appropriate model to mimic the decision-making process of human (Zsambok and Klein 1997), particularly in demanding situations. A well-known model in NDM is Klein's Recognition-Primed Decision-making (RPD) model (Klein 1998), which captures how domain experts make decisions based on the matching of past experiences and elements of the current situation. Emotion plays an important role in many aspects of the decision-making process. Many emotion models are based on the modern appraisal theory (Scherer 2005).

COSMOS is an agent-based platform of crowd simulation for military operations. In COSMOS, we incorporate emotion modeling using the appraisal theory into the RPD-based decision making process (Luo et al. 2011; Aydt et al. 2011). The experience instances of different types of agents can be specified based on various Rules of Engagement (ROE) in military scenarios. The behavior of each agent is modeled as a consequence of its decision based on the RPD framework and its emotional status in the situation. Interactions among these agents lead to the emergence of crowd-level behavior, which is hard to predict and forms the non-linear dynamics in the simulation. In this paper, a simulation model based on COSMOS is developed for specific crowd control scenarios in military operations.

Evolutionary computation (EC) techniques are non-deterministic search algorithms driven by the objectives of the searching process (Fogel 2005). EC techniques can be used to adaptively evolve a simulation model through automating different combinations of model parameter values and evaluating the simulation outcomes in order to generate the fittest candidate solutions over iterations. Evolutionary Algorithms (EAs) are typical EC techniques that have been widely applied to both numerical and combinatorial optimization problems. It has been proven highly efficient due to its fast convergency in the search space within constrained computing budget compared to the traditional sampling based data farming techniques (Decraene et al. 2010). EAs have been extended to address Pareto-based multi-objective optimization problems (Deb 2001). This is relevant for military operations as they are often characterized with multiple and conflicting objectives.

ECs were previously applied to various simplified military case studies to automatically assess the vulnerability of operational plans (e.g., (Choo et al. 2007)). Note that these case studies involve confrontations through combats or competitions, where lethal and non-lethal weapons can be assessed for their operational effectiveness. Nowadays, peace keeping efforts in urban environments are more frequently encountered (Grieger 2004). Crowd control through emotional influence without using life threatening operational plans is critical for MOUT scenarios. Such studies are lacking and have been hardly considered in the literature. We address this gap and try to achieve effective crowd control strategy in MOUT scenarios through adaptively evolving some model parameters of soldier agents in a COSMOS model. We then analyze the resulting behaviors of soldiers exhibited in the optimal solutions and to identify certain strategies that soldiers can perform in similar crowd control missions in real military operations. To assist this research, we utilize a modular evolutionary framework called CASE (Decraene et al. 2010).

This paper is organized as follows: Section 2 reviews the COSMOS platform and the CASE framework as well as the simulation model developed for the scenario used in this paper. Experiments and results are illustrated and discussed in Section 3. Section 4 concludes the paper and suggests recommendations for future work. 


\section{Hu, Decraene, and Cai}

\section{SIMULATION MODEL AND EVOLVER}

\subsection{COSMOS: Crowd Simulations for Military Operations}

COSMOS was designed using an agent-based approach to simulate realistic behaviors of different types of agents under different scenarios. In this study, we consider the motion behaviors and the interactions among individual agents in the crowd. The decision-making process is modeled according to the RPD model, which assumes people make decision based on their experiences in past situations, particularly in time-critical situations such as military operations. We model the recognition of the situation through situation assessment. The perceived situation cues and agents' emotional status affect the experience recognition. The decision-making of an agent is modeled through an experience matching process. A course of actions is carried out by an agent through the experience execution process in stages. Readers are referred to (Luo et al. 2011) for technical details of the platform.

Emotion plays an important role in the cognitive process that affects human behaviors (Scherer 2005), and thus should be considered in military crowd control operations. Existing studies have shown that angry crowds are more difficult to control and their behaviors are more acute and thus likely to conduct some destructive actions to the public (Grieger 2004). To study efficient crowd control strategy in such military operations, it is therefore necessary to incorporate the influences of emotions of both crowds and the military forces in the simulation model (Schmidt and Schneider 2004; Schwarz and Mosler 2005).

In COSMOS, we account for emotion in the overall decision making process and particularly of its influence on the recognition process. Emotion is modeled based on the appraisal emotion theory. When perceiving different emotional triggers, or appraisals, different emotional statuses are influenced accordingly. Subject to different traits of the agent, the influence on a particular emotional status is different. For example, if an agent is instigated, his emotional status of anger is likely to increase. However, individuals may react differently to the same emotional trigger due to their unique susceptibilities to this appraisal. Emotional status also changes over time. Unique personal traits also give rise to different decay rates (i.e., half-life) of an emotional status. For example, the decay for anger is faster for a peaceful person than a violent one. Emotional triggers perceived by an agent could also adjust the decay rate of the emotional status. For instance, when interacting with "pacifying" soldier agents, the anger level of a crowd may reduce faster than usual. The overall emotion system in COSMOS is shown in Fig. 1.

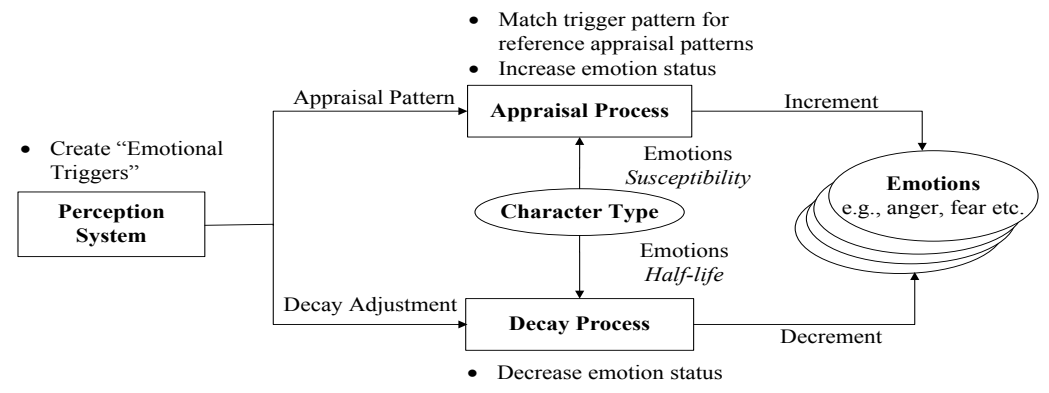

Figure 1: Appraisal-Based Emotion Model in COSMOS.

\subsection{CASE: Complex Adaptive System Evolver}

CASE is a recently developed framework which enables one to optimize simulation models through artificial evolution. There are three main components in CASE.

The model generator takes as inputs a base simulation model specified in the eXtended Markup Language (XML) and a set of model specification text files. According to these inputs, new XML simulation models are generated and sent to the simulation engine for evaluation. 


\section{Hu, Decraene, and Cai}

The simulation engine is used to execute the XML simulation models. Each simulation model is typically replicated a number of times to account for statistical fluctuations. A set of result files detailing the outcomes of the simulations (e.g., number of protester agents breaking through a guarded building entrance, number of soldiers deployed) are generated. These measurements are used to evaluate the goodness of generated models (i.e., they are the fitness (or cost) values utilized by the evolutionary algorithm).

The evolutionary algorithm receives the set of simulation results and associated model specification files, then processes the results and produces a new "generation" of model specification files. The generation of these new model specifications is driven by the user-specified objectives (e.g., minimizing the number of protester agents breaking through the entrance, minimizing the number of soldiers deployed). The algorithm iteratively generates model specifications through evolutionary search which would incrementally improve simulation results according to the objectives.

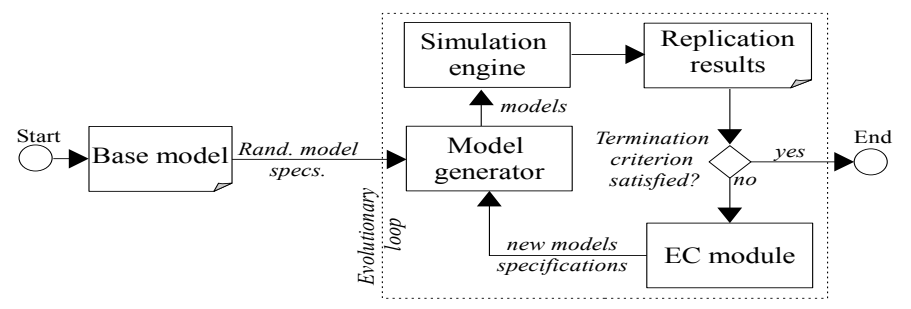

Figure 2: Components and Structure of CASE.

The overall structure of CASE is shown in Fig. 2. Based on the modular design of CASE as shown in the figure, it can be applied to evolve any simulation model (encoded in XML format). During the initialization phase, a population of simulation models with random model parameters is first created. Through the evolutionary process, the values for the evolvable parameters will be automatically adjusted and new candidate solutions (simulation models) will be evaluated according to the search objectives. Eventually, a set of evolved candidate solutions that generate the best simulation outcomes according to the objectives is obtained. CASE currently supports up to three independent objectives.

\subsection{Simulation Model}

Crowd control is often involved in MOUT scenarios, where military forces confront civilian crowds for specific purposes such as peace keeping, facility protection etc. The use of weapons, including the non-lethal ones are suggested to yield negative impact as they may spark the crowd to be even more difficult to control (Grieger 2004). Military forces can affect the overall emotional status through commands and operations such as "pacification". For example, when detecting angry civilians, soldiers can approach them and try to contain their anger level through some interactions. This process is modeled as the "pacifying" actions performed by the soldiers.

We consider a building protection scenario in the MOUT as depicted in Fig. 3(a). This scenario may represent a key facility (e.g., a government building) being guarded by military forces in real life situations. In this scenario, there are three layered ranges outside the entrance of the building to indicate the precautionary area, the alert area and the critical area. Fig. 3(b) shows the scenario in COSMOS simulation.

Three types of agents are modeled to represent protesters (i.e., the agents in black color in Fig. 3), instigators (in red color) and the soldiers (in blue color). Specific experience instances for each type of agents under the RPD framework of COSMOS are designed based on corresponding ROE in such a scenario. We discuss them as follows.

Protesters are involved in the environment with different behaviors. Initially, these agents are protesting at the open area in front of the building "peacefully". In terms of motion behavior, they randomly move around during this stage. Note that each experience set is modeled through stages under the RPD framework 


\section{Hu, Decraene, and Cai}

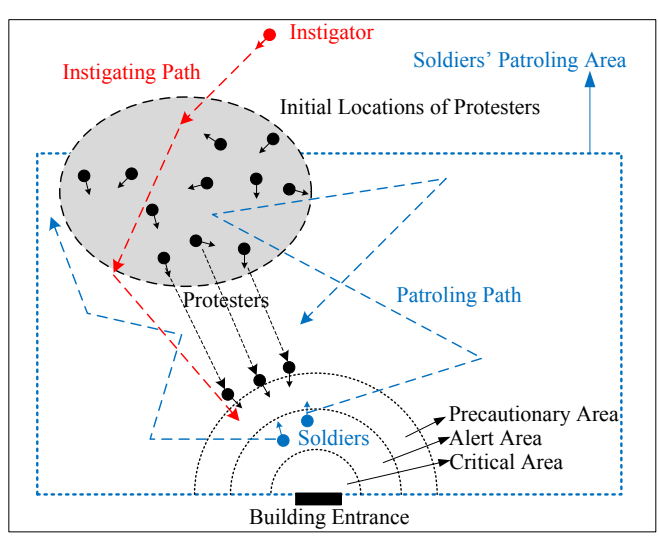

(a) Depicted Scenario

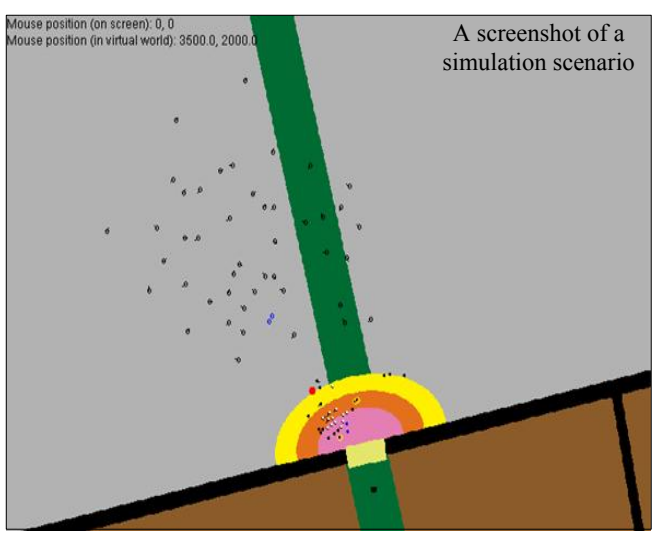

(b) Scenario in COSMOS Simulation

Figure 3: Crowd Control Simulation Scenario.

and protesters are initialized within the dotted circle at the left upper corner in the scenario as shown in Fig. 3(a). As their anger level increases by different emotional triggers, the crowd turns its random movement into a directional one heading towards the building entrance, which leads to another behavioral stage. At this stage, a protester agent determines whether or not to proceed to the next level of the sensitivity areas based on two criteria: the aggregation level in terms of the number of other surrounding protesters near this protester and the average anger level of these protesters. As shown in Table 1(a), the threshold values for the average anger are designed to increase from the outer precautionary area (0.7) to the inner critical area $(0.95)$. Such values are set through careful calibration process to generate realistic crowd behaviors in the scenario. These values reflect the protesters' behaviors naturally as they are more likely to break through the building entrance guarded by soldiers when they become angrier. The aggregation threshold decreases from precautionary area (20) to the critical area (8) because of the convergence of the area in front of the entrance.

Several emotional triggers increasing the anger of protesters are considered. First, a protester's anger increases if he is instigated by a nearby instigator. This emotional trigger of instigation forms the principle source of "angriness" in this scenario. Second, protesters can be influenced by their surrounding protesting agents, thus anger can spread and propagate through the crowd. The protesters' anger decreases regressively along a specified period of time, which differs according to different emotional types of the agent (i.e., different half-life values are specified for peaceful, normal and violent agents as shown in Table 1(b)). Additional emotional triggers may adjust and accelerate the anger decrement. In this scenario, soldiers' pacifying operation is one of such triggers.

Table 1: Emotional and Behavioral Parameter Value Settings for a Protester Agent.

(a) Behavioral Parameters

\begin{tabular}{|c|c|c|}
\hline Sensitive Area & Anger Threshold & Aggregation \\
\hline Precautionary & 0.7 & 20 \\
\hline Alert & 0.85 & 15 \\
\hline Critical & 0.95 & 8 \\
\hline
\end{tabular}

(b) Emotional Parameters (related to Anger)

\begin{tabular}{|c|c|c|}
\hline Type & Susceptibility & Half-life \\
\hline Peaceful & 0.02 & $45 s$ \\
\hline Normal & 0.05 & $60 s$ \\
\hline Violent & 0.1 & $80 s$ \\
\hline
\end{tabular}

Instigators are the initiators of turmoils among the crowd in such scenario. Instigator agents move around the crowd to instigate protesters making them angry deliberately according to a specified path. Instigator are used as a source of emotional triggers for the crowd only, i.e., we do not consider the change of emotions and behaviors of instigators themselves. 


\section{Hu, Decraene, and Cai}

Soldiers are deployed to control the crowd in order to prevent them from eventually breaking through the building entrance. The soldiers are not equipped with any weapons other than protection gears. Soldier agents essentially attempt to control the crowd through commands and pacifying actions to affect their emotional status. They patrol according to specific paths, each of which consists of a fixed number of waypoints within the soldiers' patrol area as indicated by the dashed box in Fig. 3(a).

We model the experience of soldier agents through their fear emotion and its correlation with behaviors of the soldiers. If the fear level of a soldier goes beyond a certain threshold level, then this soldier stops pacifying the crowd. This fear level increases when a soldier perceives the danger caused by a large number of surrounding protester agents. Similar to the anger of protester agents, soldiers' fear decreases over a period of time as specified by the half-life time. Emotional triggers can also affect fear decay. For example, when a fearful soldier finds backup from another soldier nearby, his fear level decreases faster. This soldier may resume to conduct his pacifying operation in the crowd once his fear level drops back to certain level.

The "gear" level of a soldier agent is modeled as an enumerate type of parameter with two possible values, "HIGH" and "LOW". In order to avoid dominating effect of a particular value on the simulation outcomes (i.e., models with deployed soldiers with "HIGH" gear always outperform models with deployed soldiers with "LOW" gear or vise versa), some constraints are considered. These constraints are likely involved in a real life scenario. As shown in Table 2, three parameters are affected by the value of a soldier agent's gear level: its susceptibility to fear, its half-life for fear decay and its maximum speed in patrolling.

Table 2: Parameter Value Settings Related to the "Gear" Level of a Soldier Agent.

\begin{tabular}{|c|c|c|c|c|}
\hline Gear Level & Susceptibility (to fear) & Half-Life (of fear) & Maximum Speed & Fear Threshold \\
\hline "HIGH" & 0.2 & $60 s$ & $0.1 \mathrm{~m} / \mathrm{s}$ & 0.65 \\
\hline "LOW" & 1.0 & $30 \mathrm{~s}$ & $1.0 \mathrm{~m} / \mathrm{s}$ & 0.65 \\
\hline
\end{tabular}

Specifically, with "HIGH" gear level, a soldier agent moves much slower (with maximum speed of $0.1 \mathrm{~m} / \mathrm{s}$ ) than the one with "LOW" gear $(1 \mathrm{~m} / \mathrm{s})$. However, it is less susceptible (with susceptibility of 0.2 to fear) to the emotional triggers that will increase its fear level than the ones with "LOW" gear (1.0). In addition, the decay of fear for the agent with "HIGH" gear is longer (with half-life of 60s) than the one with "LOW" gear (30s). As aforementioned, the pacifying operation of a soldier agent can adjust decay of protesters' anger. Here, the fear of a soldier agent, in return, affects its pacifying operation. A soldier agent stops pacifying when its fear level goes beyond a threshold (0.65). These parameter settings through the calibration process aim to create complex conflicting interactions among individual agents through their emotions and behaviors in a realistic manner under this scenario.

\section{EXPERIMENTS AND RESULTS}

\subsection{Experimental Settings}

Based on the scenario specified above, we consider a Pareto-based multi-objective optimization where the number of protesters breaking through the building entrance and the number of deployed soldiers are to be minimized. In such a scenario, those two search objectives are thus conflicting with each other.

In the current experiment, 100 protesters, one instigator and a variable number (evolved between one to four) of soldier agents are involved in COSMOS simulation. The environment is initialized according to Fig. 3(a). Parameters of the soldier agents are to be evolved through CASE. Table 3 lists these parameters we account for in this study.

Table 3: Evolvable Parameters and Their Value Ranges.

\begin{tabular}{|c|c|c|}
\hline Number of Deployed Soldiers & Gear Level & Coordinates of Waypoints for Each Soldier \\
\hline numerical & enumerate & numerical \\
\hline $1 \sim 4$ & $\left\{\right.$ " $\left.H I G H^{\prime \prime}, " L O W^{\prime \prime}\right\}$ & $x \in[0,60], y \in[0,30]$ \\
\hline
\end{tabular}




\section{Hu, Decraene, and Cai}

As shown in Table 3, three types of parameters are evolved for soldier agents in the experiments: the number of deployed soldiers (range from one to four), the gear level of the soldiers ("HIGH" or "LOW") and the coordinates $(\mathrm{x}, \mathrm{y})$ of each waypoint along an evolvable path for each soldier. Note that these coordinates will be transformed to the actual coordinates in the virtual environment during simulation. In the current study, there is a fixed number (eight) of waypoints in a path with a fixed duration of 40 simulation frames for a soldier agent to stay at each waypoint. So, the size of the search space is large (more than $10^{26}$ ).

The Non-dominated Sorting Algorithm II (NSGA-II) (Deb et al. 2000) is employed to conduct the evolutionary search. This algorithm is executed using the settings as specified in Table 4. These parameter values are commonly used, as reported in the literature, when NSGA-II is applied to two-objective optimization problems (Deb et al. 2000; Decraene et al. 2010). The population size and the number of iterations indicate that 60,000 distinct COSMOS simulation models are generated and evaluated during a single experimental run. Each simulation model instance is executed/replicated 30 times to account for statistical fluctuations.

Table 4: Settings for NSGA-II Evolutionary Algorithm in the Experiments.

\begin{tabular}{|l|l|l|l|l|l|l|}
\hline Population & Generations & Replication & $\begin{array}{l}\text { Mutation } \\
\text { Prob }\end{array}$ & $\begin{array}{l}\text { Mutation } \\
\text { Index }\end{array}$ & $\begin{array}{l}\text { Cross Over } \\
\text { Rate }\end{array}$ & $\begin{array}{l}\text { Cross Over } \\
\text { Index }\end{array}$ \\
\hline 300 & 200 & 30 & 0.1 & 20 & 0.9 & 20 \\
\hline
\end{tabular}

\subsection{Results Analysis}

\subsubsection{Hypervolume Dynamics}

The hypervolume indicator measures the quality of Pareto fronts (Deb 2001). Whenever a set of solutions dominates another set, its hypervolume indicator value is always better than the one of the latter. This method is widely used in the multi-objective evolutionary computation field to assess search algorithms (Zitzler et al. 2007).

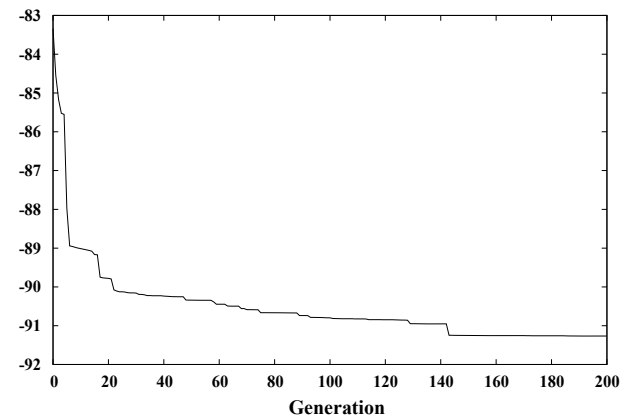

(a) Dynamics of HyperVolume Indicator Values

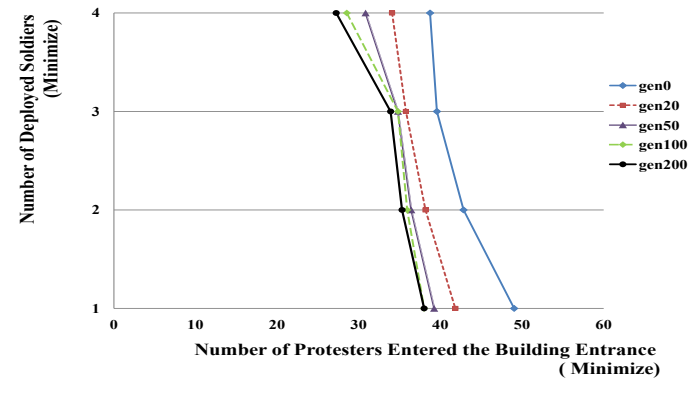

(b) Pareto Front Approximations over Evolutionary Search

Figure 4: Evolutionary Search Results from CASE.

The dynamics of the hypervolume indicator values shown in Fig. 4(a) provides information with regards to the improvement of the Pareto front over the evolutionary search. Fig. 4(b) illustrates the improvement of the Pareto front approximation overtime for five distinct generations. The hypervolume dynamics suggests that the evolutionary search may have reached a certain level of convergence. However, given the large number of parameters and the available value domain for these parameters, further improvements are potentially achievable by increasing the population size and the generations in the EA setting. 


\section{Hu, Decraene, and Cai}

\subsubsection{Emotional and Behavioral Analysis}

Taking the solutions from the Pareto front at generation 200, we examine these models by replicating them in COSMOS and to investigate the emotional and behavioral dynamics of the agents, in particular, the soldier agents through the simulation. The simulation results of the four models are summarized in Fig. 5 (emotional statistics) and Fig. 6 (paths of soldier agents).

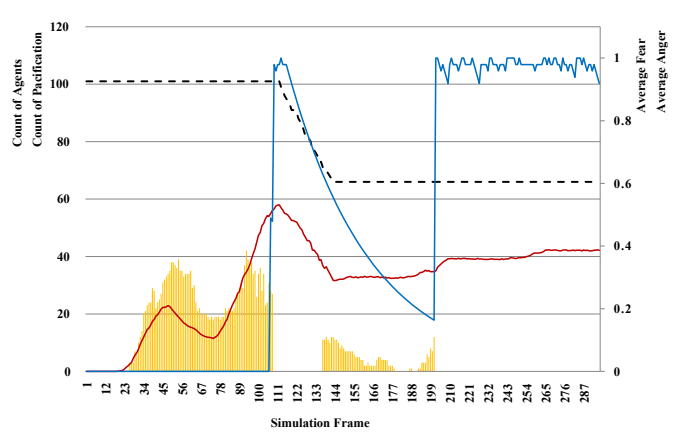

(a) One Soldier of LOW Gear

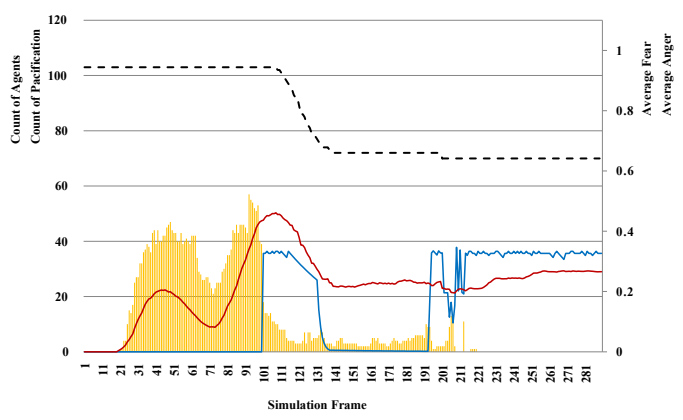

(c) Three Soldiers of LOW Gear

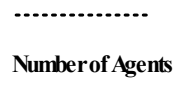

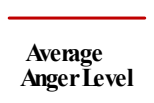

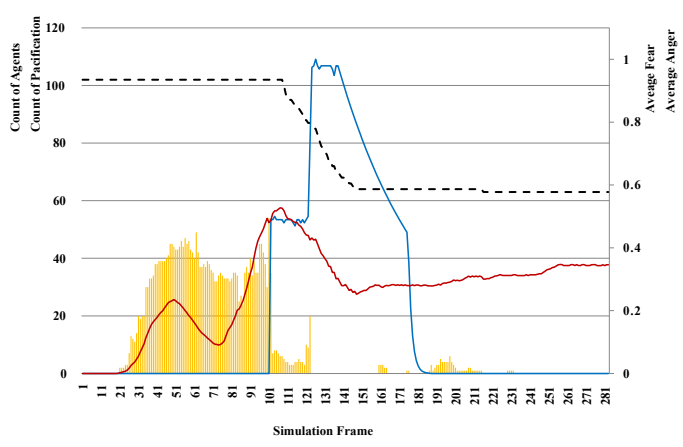

(b) Two Soldiers of LOW Gear

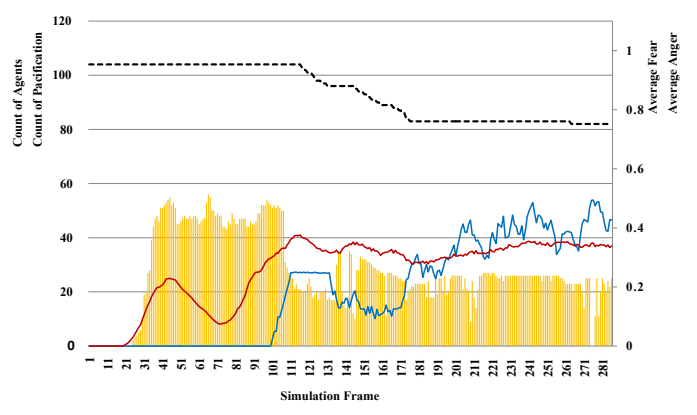

(d) Four Soldiers of HIGH Gear

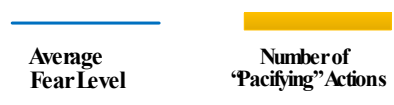

Figure 5: Simulation Statistics using the Set of Solutions from the Pareto Front (at generation 200).

From the results, we can observe that the overall crowd is better controlled in terms of the number of protesters that have entered the building entrance as the number of deployed soldiers increases in the model. This is to some extent expected when considering the conflicting multi-objectives of the study as discussed earlier. In all four solution models, the total number of "pacifying" emotional triggers on protester agents (the orange bars in Fig. 5) starts to increase significant at relative early stage of the simulation (i.e., averaging at around the 20th simulation frame). By correlating this finding with the simulation, we note that the soldier agents are initialized at positions within the protesters and near the instigators. Such a deployment provides opportunities for early interception on the crowd.

When the number of soldiers equals one, two or three, the soldier agents has "LOW" gear level. When examining in the simulation results, we observe that the soldier agents in these models move along different waypoints across wide range of areas (see Figs. 6(a), 6(b), and 6(c)). We observe consecutive waypoints with positions within and away from aggregated cluster of angry crowds. As a result, the discontinuity of their "pacifying" operations are reflected by the pulses in Figs. 5(a), 5(b), and 5(c). Due to their high susceptibility to the fear emotion and their fast speed to move away from the angry protesters, these soldier 


\section{Hu, Decraene, and Cai}

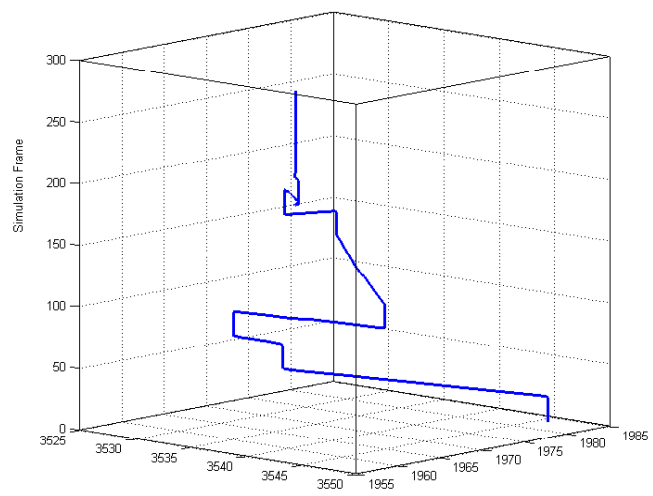

(a) One Soldier of LOW Gear

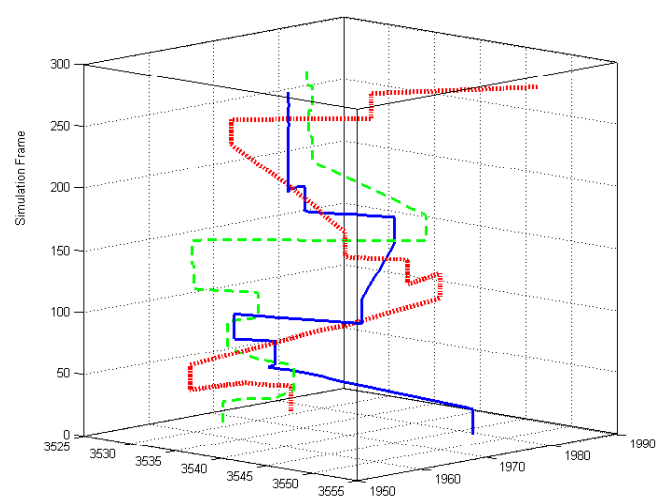

(c) Three Soldiers of LOW Gear

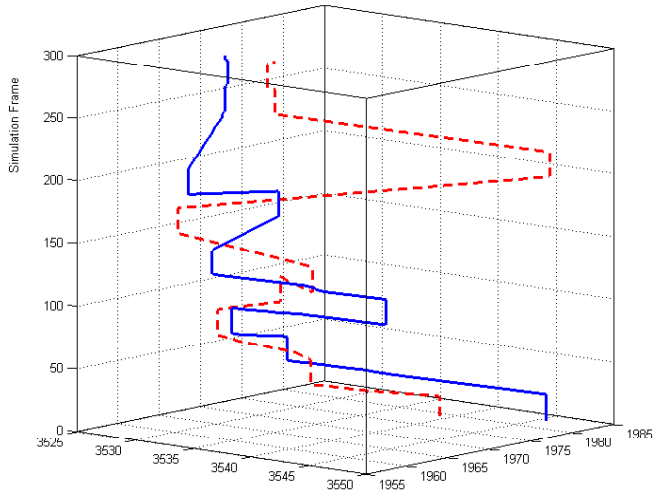

(b) Two Soldiers of LOW Gear

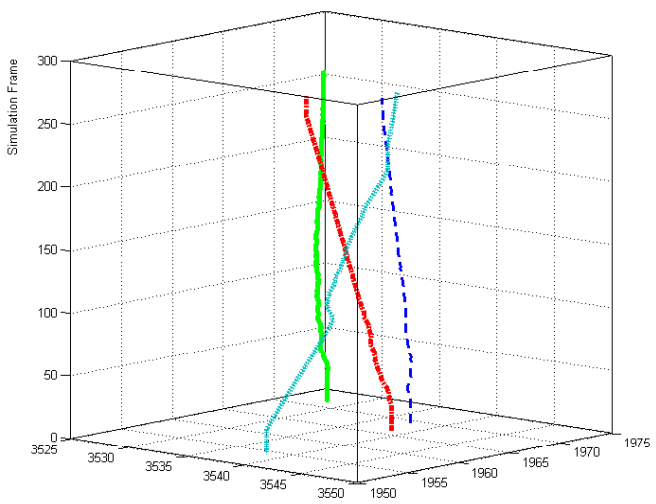

(d) Four Soldiers of HIGH Gear

Figure 6: Evolved Patrol Paths of Soldier Agents.

agents' fear level fluctuates significantly as indicated by the sharp rise and drop of the average fear level in the figures. We also note that the evolved path for at least one soldier agent in all of these models matches with the path of the instigator quite well.

It is interesting to note that when the number of soldiers increases to four (Fig. 5(d)), the gear level is changed from "LOW" to "HIGH". By examining the results in COSMOS, the four soldier agents are initially deployed in three squads with two, one and one agent(s) in each. Three of them are initiated at the positions within the initialization area of protesters away from the precautionary area as previously shown in Fig. 3(a) and one is inside the precautionary area. As the instigator moves into the crowd, three of the soldier agents confront the angry protesters as indicated by the significant increase of "pacifying" operations in Fig. 5(d). As the instigator pushes the crowd towards the building entrance, the soldier agent near the entrance move forwards to confront the angry protesters. Its fear level increases fast and it stops pacifying (frame 99 to 110 in Fig. 5(d)). The squad of two soldiers tries to move towards the precautionary area to backup the fearful soldier (Fig. 6(d)). Due to their speed limit, they reach the area of the fearful soldier around 30 frames later. As a result, the aggregated anger of protesters in front of the building entrance further increases and they start to break through the entrance during this period. This latency results in the drop of pacifying operations, the increase of protesters anger and the initial drop of the number of agents remaining in simulation from frame 104 to 135 (as shown in Fig. 5(d)). The other soldier continue to pacify the remaining protesters outside of the precautionary area. Since a large proportion of protesters confront the three soldier agents at the precautionary area, the average anger level for the protesters and the average fear level of soldiers fluctuates from frame 135 onwards. 


\section{$H u$, Decraene, and Cai}

Another interesting observation is the intersections of soldier agents' paths when multiple of them are deployed (see Figs. 6(b), 6(c), and 6(d)). Such path intersections and meeting of the soldiers affect their emotional status (and thus their pacifying operations) as reflected by the relative steep decrease of their average fear level in Fig. 5. For example, in Fig. 5(c), the average fear of soldier agents steeply reduces from frame 129 to 137. Such reduction can be attributed to the intersection of all the 3 soldier agents' paths during the same simulation frames as indicated in Fig. 6(c).

\subsubsection{Potential Insights}

The results from this preliminary study on crowd control in MOUT scenario seem appealing. From the current results, we could conclude, with cautions, the following crowd control strategies that seem effective in the corresponding peace-keeping scenarios. Early interception is important. Soldiers should try to prevent crowds aggregation and prevent anger from spreading as early as possible.

Given the trade-off between the protection and mobility of soldiers as illustrated in our study, the deployment strategy of soldiers with different protection levels should be considered. Soldiers with stronger protection is suggested to be deployed at critical area (e.g., the sensitive areas in front of the building entrance in our case) due to low susceptibility to fear and slow movement speed. Soldiers with less protection could be deployed to move among crowd to cover a larger area due to their mobility. However, these soldiers are advised to be deployed in small groups rather than individuals due to their relative vulnerability in facing threat or danger from the crowd.

The patrol paths of all the soldiers are suggested to cover a wide area including both the immediate sensitive front and the remote area occupied by the crowd. This is due to the requirement of early interception as indicated above. Soldiers should patrol along paths such that they cover some important areas, which usually are dynamic and subject to the requirements of the mission. For example, in our case, the soldiers generally move towards the area with a large crowd exhibiting a high average anger level due to the high probability of any destructive consequence in these areas. The path planning should also take advantage of the environment in the mission. In this study, the three sensitive areas form a barrier effect upon the motion of protesters. They aggregate along the boundary of an area and only proceed into the area until a higher anger level is reached. The soldiers in such cases can take the opportunity to retreat and reduce their fear level at the inner empty area in order to sustain their control on the crowd over a long period of time.

\section{CONCLUSIONS AND FUTURE WORK}

In this paper, we have proposed an approach to assist research in military operations in urban terrain (MOUT) through adaptive evolution of agent-based simulation models. We first provided a brief introduction to the key technologies supporting our research for military operations, namely the agent-based simulation platform COSMOS and the complex adaptive system evolver CASE. A typical scenario of MOUT, building protection, was examined to demonstrate the potential of our approach to find effective crowd control strategies, where different types of agents interact in a complex manner through their emotions and behaviors. Two objectives were proposed for the evolutionary algorithm to search for optimal solutions (i.e., models with specific parameters). We conducted experiments examining the soldier agents' operational plans. Several insights have been identified that may potentially lead to the identification of more efficient crowd control strategies. These results suggest that our approach is promising in assisting research in military operations.

To further develop this work, we plan to reduce the evolvable parameter space. The path evolutionary problems discussed in (Decraene et al. 2010) could provide some heuristics to refine the evolvable parameter design to reduce the number of waypoints in specifying soldier agents' patrol paths. In addition, co-evolutionary computation may also be adopted to further investigate the optimal solutions in even more dynamic and competitive scenarios, where interactions among different types of agents are continuously changing in run-time. For example, in viewing of soldiers nearby, the instigators may react and change 


\section{Hu, Decraene, and Cai}

their instigation paths. This future work may extend our approach to more military operations, planning and training applications.

\section{ACKNOWLEDGMENTS}

We would like to thank the Defence Research and Technology Office, Ministry of Defence, Singapore for supporting the Evolutionary Computing Based Methodologies for Modeling, Simulation and Analysis project under the project agreement POD0814214.

\section{REFERENCES}

Aydt, H., M. Lees, L. Luo, W. Cai, M. Low, and S. Kadirvelen. 2011, August. "A Computational Model of Emotions for Agent-Based Crowds in Serious Games". In Web Intelligence and Intelligent Agent Technology (WI-IAT), 2011 IEEE/WIC/ACM International Conference on, edited by J. F. Hübner, J.-M. Petit, and E. Suzuki, Volume 2, $72-80$.

Bonabeau, E. 2002, May. "Agent-based modeling: Methods and techniques for simulating human systems". Proceedings of the National Academy of Sciences 99 (3): 7280-7287.

Choo, C. S., C. L. Chua, and S.-H. V. Tay. 2007. "Automated Red Teaming: A Proposed Framework for Military Application". In Proceedings of the 9th annual conference on Genetic and evolutionary computation, edited by D. Thierens, H.-G. Beyer, J. Bongard, J. Branke, J. A. Clark, D. Cliff, C. B. Congdon, K. Deb, B. Doerr, T. Kovacs, S. Kumar, J. F. Miller, J. Moore, F. Neumann, M. Pelikan, R. Poli, K. Sastry, K. O. Stanley, T. Stutzle, R. A. Watson, and I. Wegener, GECCO '07, 1936-1942. New York, NY, USA: ACM.

Deb, K. 2001, June. Multi-Objective Optimization Using Evolutionary Algorithms. first ed. Wiley.

Deb, K., S. Agrawal, A. Pratap, and T. Meyarivan. 2000. "A Fast Elitist Non-dominated Sorting Genetic Algorithm for Multi-objective Optimization: NSGA-II”. In Parallel Problem Solving from Nature PPSN VI, edited by M. Schoenauer, K. Deb, G. Rudolph, X. Yao, E. Lutton, J. Merelo, and H.-P. Schwefel, Volume 1917 of Lecture Notes in Computer Science, 849-858. Springer Berlin / Heidelberg.

Decraene, J., M. Chandramohan, M. Low, and C. Choo. 2010, December. "Evolvable Simulations applied to Automated Red Teaming: A Preliminary Study". In Proceedings of the 2010 Winter Simulation Conference, edited by B. Johansson, S. Jain, J. Montoya-Torres, J. Hugan, and E. Yücesan, 1444-1455. Piscataway, New Jersey: Institute of Electrical and Electronics Engineers, Inc.

Decraene, J., M. Low, F. Zeng, S. Zhou, and W. Cai. 2010. "Automated Modeling and Analysis of Agentbased Simulations using the CASE Framework". In The 11th International Conference on Control Automation Robotics \& Vision (ICARCV 2010), edited by C. Wen, P. N. Suganthan, and E. Sung, 346-351. Singapore: IEEE.

Decraene, J., F. Zeng, M. Y. H. Low, S. Zhou, and W. Cai. 2010. "Research Advances in Automated Red Teaming”. In Proceedings of the 2010 Spring Simulation Multiconference, 145-152. Orlando, FL, USA: Society for Computer Simulation International.

Desch, M. C. (Ed.) 2001. Soldiers in Cities: Military Operations on Urban Terrain. Army War College (U.S.). Strategic Studies Institute.

Fogel, D. B. 2005, December. Evolutionary Computation: Toward a New Philosophy of Machine Intelligence. Third ed. IEEE Press Series on Computational Intelligence. Wiley-IEEE Press.

Grieger, D. 2004. "An Overview of Crowd Control Theory and Considerations for the Employment of Non-Lethal Weapons". Technical Report DSTO-GD-0373, Land Operations Division System Sciences Laboratory, Australian Government, DSTO, Australia.

Klein, G. A. 1998. Sources of Power: How People Make Decisions. Cambridge, MA: MIT Press.

Laclavk, M., t. Dlugolinsk, M. Łeleng, M. Kvassay, B. Schneider, H. Bracker, M. Wrzeszcz, J. Kitowski, and L. Hluch. 2012. "Agent-Based Simulation Platform Evaluation in the Context of Human Behavior Modeling”. In Advanced Agent Technology, edited by F. Dechesne, H. Hattori, A. ter Mors, J. Such, 


\section{Hu, Decraene, and Cai}

D. Weyns, and F. Dignum, Volume 7068 of Lecture Notes in Computer Science, 396-410. Springer Berlin / Heidelberg.

Larson, R. 1981. Urban Operations Research. Prentice Hall.

Luo, L., S. Zhou, W. Cai, M. Lees, M. Y.-H. Low, and K. Sornum. 2011. "HumDPM: A Decision Process Model for Modeling Human-Like Behaviors in Time-Critical and Uncertain Situations". Transactions on Computational Science 12:206-230.

Scherer, K. R. 2005. Appraisal Theory, Chapter 30, 637-663. Wiley.

Schmidt, B., and B. Schneider. 2004. "The Reflective Control of Cognition and Emotion". In Proceedings of the 2004 Operational Research Society Simulation Workshop, edited by S. C. Brailsford, L. Oakshott, S. Robinson, and S. J. E. Taylor, 145-154. Birmingham, UK.

Schwarz, G., and H.-J. Mosler. 2005. "Investigating Escalation Processes in Peace Support Operations: An Agent-based Model about Collective Aggression". In Proceedings of the 3rd Annual Conference of the European Social Simulation Association (ESSA 2005), edited by K. Troitzsch, 191-197. Koblenz, Germany.

Zitzler, E., D. Brockhoff, and L. Thiele. 2007. "The Hypervolume Indicator Revisited: On the Design of Pareto-compliant Indicators Via Weighted Integration". In Evolutionary Multi-Criterion Optimization, edited by S. Obayashi, K. Deb, C. Poloni, T. Hiroyasu, and T. Murata, Volume 4403 of Lecture Notes in Computer Science, 862-876. Springer Berlin / Heidelberg.

Zsambok, C. E., and G. A. Klein. 1997. Naturalistic Decision Making. New Jersey: Lawrence Erlbaum Associates.

\section{AUTHOR BIOGRAPHIES}

NAN HU is a research Project Officer in the School of Computer Engineering (SCE) at Nanyang Technological University(NTU), Singapore. He received his Bachelor of Computer Engineering with Honor and a Minor in Psychology from NTU in 2007. He is a final year part-time Ph.D candidate in the school. His research interests include Agent-based Modeling \& Simulation, Complex Systems, Human Cognitive Process, Perception, Decision-making and Behavioral Models. His Ph.D focus is on the Spatial-Temporal Patterns and Pedestrian Simulations.

JAMES DECRAENE is a Scientist in the Institute of High Performance Computing, Agency for Science, Technology and Research (A*Star). He was previously a postdoctoral research fellow in the School of Computer Engineering at Nanyang Technological University (NTU), Singapore. He received his Bachelor of Computer Science with First Class Honor from Teesside University, UK in 2004. In 2009, he completed his Ph.D. in Complex Adaptive Systems at the School of Electronic Engineering, Dublin City University, Ireland. His research interests include Complex Adaptive Systems, Agent-based Modeling \& Simulation, Evolutionary Computation and High Performance Computing. His work aims at engineering more efficient and robust complex artificial systems through simulation-based optimization and high performance computing.

WENTONG CAI is a Professor in the School of Computer Engineering at Nanyang Technological University, Singapore. He is also the Director of the Parallel and Distributed Computing Centre. He received his Ph.D. in Computer Science from University of Exeter (UK) in 1991. His expertise is mainly in the areas of Modeling and Simulation (particularly, modeling and simulation of large-scale complex systems, and system support for distributed simulation and virtual environments) and Parallel and Distributed Computing (particularly, Cloud, Grid and Cluster computing). He is an associate editor of the ACM Transactions on Modeling and Computer Simulation (TOMACS) and an editor of the Future Generation Computer Systems (FGCS). 